\title{
Answer to the Letter to the Editor of H. Namazi concerning "Denosumab: a potential new and innovative treatment option for aneurysmal bone cysts" by Lange T, Stehling C, Fröhlich B, Klingenhöfer M, Kunkel P, Schneppenheim R, Escherich G, Gosheger G, Hardes J, Jürgens H, Schulte TL (2013), Eur Spine J;22(6):1417-1422
}

\author{
T. Lange $\cdot$ T. L. Schulte
}

Received: 3 August 2013/Revised: 3 August 2013/Accepted: 3 August 2013/Published online: 23 August 2013

(C) Springer-Verlag Berlin Heidelberg 2013

Dear Editor,

We read with great interest and appreciate the comment on an additional route how Denosumab works [1]. Our article focused on the clinical application of Denosumab in aneurysmal bone cysts [2]. This comment highlights the physiological mechanisms of the effect of Denosumab and thus perfectly completes and complements our work. In general, the continuous process of bone resorption and formation underlies different mechanisms and is well balanced in the healthy skeleton. In addition to the RANKL mechanism, the Wnt/B-catenin pathway has been identified to play an important role in bone formation [3]. Long-term treatment with Denosumab has been shown to increase sclerostin levels which has been proven to lead to a depressed bone formation [4], but simultaneously DKK1 decreases which is thought to inhibit the sclerostin effect [1]. The clinical effect of Denosumab also in children will be a compound of these different pathways.

Conflict of interest None.

\section{References}

1. Gatti D, Viapiana O, Fracassi E, Idolazzi L, Dartizio C, Povino MR et al (2012) Sclerostin and DKK1 in postmenopausal osteoporosis treated with denosumab. J Bone Miner Res 27(11): 2259-2263

2. Lange T, Stehling C, Fröhlich B, Klingenhöfer M, Kunkel P, Schneppenheim R, Escherich G, Gosheger G, Hardes J, Jürgens H, Schulte TL (2013) Denosumab: a potential new and innovative treatment option for aneurysmal bone cysts. Eur Spine J 22(6): $1417-1422$

3. Baron R, Rawadi G (2007) Targeting the Wnt/beta-catenin pathway to regulate bone formation in the adult skeleton. Endocrinology 148:2635-2643

4. Gatti D, Viapiana O, Idolazzi L, Fracassi E, Rossini M, Adami S (2011) The waning of teriparatide effect on bone formation markers in postmenopausal osteoporosis is associated with increasing serum levels of DKK1. J Clin Endocrinol Metab 96(5): 1555-1559
T. Lange · T. L. Schulte $(\square)$

Department of Orthopedics and Tumor Orthopedics,

Münster University Hospital, Albert-Schweitzer-Campus 1,

48149 Münster, Germany

e-mail: schultet@ukmuenster.de

T. Lange

e-mail: tobias.lange@ukmuenster.de 